\title{
Testing of the Maximal Dynamic Output Hypothesis in Trained and UnTrained SubJects
}

\author{
James L. Nuzzo, ${ }^{1}$ Jeffrey M. McBride, ${ }^{2}$ Andrea M. Dayne, ${ }^{2}$ Michael A. Israetel, ${ }^{2}$ \\ Charles L. Dumke, ${ }^{3}$ and N. Travis Triplett ${ }^{2}$ \\ ${ }^{1}$ Department of Exercise and Rehabilitative Sciences, Slippery Rock University, Slippery Rock, Pennsylvania; \\ ${ }^{2}$ Department of Health, Leisure and Exercise Science, Appalachian State University, Boone, North Carolina; and \\ ${ }^{3}$ Department of Health and Human Performance, The University of Montana-Missoula, Missoula, Montana
}

\begin{abstract}
Nuzzo, JL, McBride, JM, Dayne, AM, Israetel, MA, Dumke, CL, and Triplett, NT. Testing of the maximal dynamic output hypothesis in trained and untrained subjects. J Strength Cond Res 24(5): 1269-1276, 2010-The maximal dynamic output (MDO) hypothesis is a newly proposed concept, which suggests that the muscular system of the lower limbs is designed to produce maximal power output when performing countermovement vertical jumping (CMJ) at body mass as opposed to other loading conditions. However, it is unclear if the MDO concept can be applied to individuals with different levels of maximal strength. The purpose of this investigation was to determine if subjects, who have distinct differences in maximal strength, maximize CMJ power at body mass. Fourteen male strength-power trained subjects (squat 1 repetition maximum (1RM)-to-body mass ratio $=1.96 \pm 0.24$ ) and 6 untrained male subjects (squat $1 \mathrm{RM}$-to-body mass ratio $=$ $0.94 \pm 0.18$ ) completed CMJs with loads that were less than, equal to, and greater than body mass. Loads less than body mass were accomplished with a custom-designed unloading apparatus, and loads greater than body mass were accomplished with a barbell and weights. In both groups, mean values for CMJ peak and mean power were greatest during the body mass jump. Power outputs at body mass were significantly different $(p \leq 0.05)$ than power outputs at various conditions of loading and unloading. These data support the MDO hypothesis and its application to individuals with significantly different 1RM-to-body mass ratios. Additionally, these data further support the idea that body mass CMJs are a theoretically sound way to train for power because of the maximal power outputs that are produced during this condition.
\end{abstract}

KEY WoRDS strength, power, vertical jump, unloading

Address correspondence to James L. Nuzzo, james.nuzzo@sru.edu.

24(5)/1269-1276

Journal of Strength and Conditioning Research

(C) 2010 National Strength and Conditioning Association

\section{INTRODUCTION}

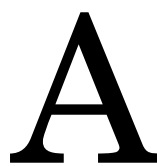

debate exists as to which load maximizes power output during countermovement vertical jumping (CMJ) $(1-3,7,8,15,16,20)$. The results from previous studies have been conflicting as loads ranging from $0 \%$ (body mass) $(3,7,8,15,16)$ to $59 \%$ (2) of a 1 repetition maximum (1RM) back squat have been found to maximize $\mathrm{CMJ}$ power output (i.e., the optimal load for power). The conflicting results regarding the load that maximizes $\mathrm{CMJ}$ power output appear to be primarily the consequence of the different data-collection techniques used $(4,5,9,11)$. Comprehensive investigations that have assessed the effects of these different data-collection techniques on $\mathrm{CMJ}$ power output have concluded that the most valid way to assess CMJ power is by using a combination of a force plate and position transducers $(4,5)$. Thus, because of methodological flaws in data-collection techniques, some studies may have misrepresented power output at various loading conditions and may not have correctly identified the optimal load for power. When valid measurement techniques have been used, the load that maximizes CMJ power output has been found to be at body mass $(3,7,8,15,16)$.

As a result of the accumulation of evidence that suggests that the optimal load for CMJ power is at body mass, Jaric and Markovic (13) have developed the maximum dynamic output (MDO) hypothesis that suggests that "the muscular system of the lower limbs is designed to produce maximum dynamic output in rapid movements when loaded only by the weight and the inertia of its own body." The evidence for this hypothesis is particularly strengthened by studies that have not only loaded, but also unloaded subjects, to examine a more comprehensive load-power relationship for the $\mathrm{CMJ}$ $(3,15)$. Previous research that has implemented unloading, however, has only assessed moderately active participants $(3,15)$. As indicated by Jaric and Markovic (13), it is still unclear whether or not the MDO hypothesis applies to all populations, especially when considering previous findings that have suggested that levels of lower-body maximal strength may affect the load that maximizes power output $(1,20)$. Stone et al. $(20)$ have reported that subjects with back squat 1RM-to-body mass ratios of 1.21 produced peak power 
at the lightest load studied (i.e., 10\% of 1RM), whereas stronger individuals with 1RM-to-body mass ratios of 2.00 produced peak power at a load equal to $40 \%$ of $1 \mathrm{RM}$. On the other hand, Baker (1) discovered that stronger athletes exhibited greater power outputs at lighter loads when compared with subjects with lower levels of maximal strength. Thus, further investigation, using valid measurement techniques, is necessary to clarify the effect of lowerbody maximal strength on the CMJ load-power relationship and to determine if the MDO hypothesis can be applied to individuals with different levels of lower-body maximal strength. Thus, the purpose of this study was to test the MDO hypothesis in trained and untrained subjects and determine if levels of maximal strength affect the load that maximizes power output.

\section{Methods}

\section{Experimental Approach to the Problem}

To determine if levels of maximal strength affect the load that maximizes power output, 2 distinct subject groups, strengthpower trained subjects with high 1RM-to-body mass ratios, and untrained subjects with low 1RM-to-body mass ratios, participated in 2 testing sessions that were separated by 7 days. The first session included measurements of body height, body weight, and body fat percentage based on skinfolds from 3 sites (chest, abdomen, and thigh) $(12,19)$. In addition, the first session included a maximal CMJ height test, a back squat 1RM assessment, and several familiarization trials with CMJs using an unloading apparatus. The second testing session required subjects to complete CMJs at various loads in a randomized order. Mean power and peak power were measured during the CMJs across the loading and unloading spectrum to test for the MDO.

\section{Subjects}

Fourteen strength-power trained and 6 untrained male subjects participated in this investigation (Table 1). For inclusion into the study, strength-power trained subjects were required to have at least 2 years of previous strength and power training. This group of subjects included both recreational athletes and competitive athletes who had backgrounds in baseball, basketball, volleyball, track and field sprinting and jumping events, powerlifting, and Olympic weightlifting. All strength-power trained subjects were assessed during the offseason for their respective sports. Furthermore, all strengthpower trained subjects had a back squat 1RM-to-body mass ratio $\geq 1.79$ and a $C M J$ height $\geq 50 \mathrm{~cm}$. Untrained subjects were required to have little or no past experiences in strength or power training in the past 2 years. All untrained subjects had a 1RM-to-body mass ratio between 0.70 and 1.20 and a $\mathrm{CMJ}$ height $\leq 43 \mathrm{~cm}$. Before testing, all subjects were informed of the study procedures and were required to sign an informed consent. Approval from the Appalachian State University Institutional Review Board was obtained before the start of the investigation.

\section{Procedures}

Maximal Strength Testing. Back squat 1RM was assessed after an appropriate warm-up protocol (17). The warm-up protocol consisted of multiple repetitions at loads equal to 30\% (8-10 repetitions), 50\% (4-6 repetitions), 70\% (2-4 repetitions), and $90 \%$ (1 repetition) of the subject's estimated $1 \mathrm{RM}$. During all attempts, subjects were required to lower the bar to a point where an $80^{\circ}$ knee angle was attained. Before the start of the warm-up, a goniometer was used to visually demonstrate the attainment of an $80^{\circ}$ knee angle while squatting. Subjects were given up to 4 maximal attempts to achieve a $1 \mathrm{RM}$. Rest periods of 3-5 minutes were given between trials. Because untrained subjects did not have a previous estimation of their maximal strength capabilities, a load equal to $1.0 \times$ body mass was used as an estimated 1RM. For the untrained subjects, any necessary modifications in warm-up loads were made by a test administrator.

Vertical Jump Testing. Subjects completed CMJs at 7 different loads in a randomized order. Three maximal effort CMJs were performed at each loading and unloading condition. Subjects were given a 1-minute rest period between jump trials and were given a 3 -minute rest period between loading and unloading conditions. The depth of the countermovement during the CMJs was self-selected by the subjects.

Load Determination. The 7 loads used in CMJ testing were determined as a percentage of maximal dynamic strength (MDS) (7). The calculation of MDS (MDS $=1 \mathrm{RM}+[$ Body Mass - Shank Mass]) is an appropriate method for CMJ load determination because it accounts for the external load lifted during the $1 \mathrm{RM}$ test in addition to the individual body mass

TABLE 1. Subject characteristics for the trained $(n=14)$ and untrained $(n=6)$ groups.

\begin{tabular}{lcc}
\hline & Trained & Untrained \\
\hline Age $(\mathrm{y})$ & $21.5 \pm 1.4$ & $20.0 \pm 1.7$ \\
Height $(\mathrm{cm})$ & $177.7 \pm 5.4$ & $174.8 \pm 9.2$ \\
Body mass $(\mathrm{kg})$ & $85.7 \pm 10.6$ & $74.6 \pm 19.3$ \\
Body fat $(\%)^{*}$ & $8.6 \pm 2.9$ & $14.4 \pm 6.0$ \\
Fat mass $(\mathrm{kg})$ & $7.6 \pm 3.1$ & $11.5 \pm 7.1$ \\
Fat-free mass (kg)* & $78.1 \pm 8.2$ & $63.0 \pm 12.9$ \\
CMJ height $(\mathrm{m})^{*}$ & $0.55 \pm 0.03$ & $0.37 \pm 0.04$ \\
Squat 1RM (kg)* & $167.9 \pm 27.8$ & $68.5 \pm 12.7$ \\
$\begin{array}{l}\text { Squat 1RM-to-body } \\
\quad \text { mass ratio* }\end{array}$ & $1.96 \pm 0.24$ & $0.94 \pm 0.18$ \\
Maximal dynamic & $243.3 \pm 34.9$ & $134.1 \pm 26.9$ \\
$\quad$ strength (kg)* & &
\end{tabular}

Values reported as mean $\pm S D$.

$\mathrm{CMJ}=$ countermovement jump; $1 \mathrm{RM}=1$-repetition maximum.

*Significant difference $(p \leq 0.05)$ between trained and untrained groups. 
TABLE 2. CMJ loading and unloading conditions for the trained group.

\begin{tabular}{lcccrr}
\hline & Un/Ext mass $(\mathrm{kg})$ & CMJ load $(\mathrm{kg})$ & BW & \%1RM & Unload force (N) \\
\hline $10 \%$ MDS & $-51.1 \pm 6.8$ & $24.3 \pm 3.5$ & $0.28 \pm 0.02$ & $-31.0 \pm 4.2$ & $439.0 \pm 44.2$ \\
$20 \%$ MDS & $-26.7 \pm 5.4$ & $48.7 \pm 7.0$ & $0.57 \pm 0.05$ & $-16.4 \pm 3.8$ & $286.4 \pm 45.6$ \\
$30 \%$ MDS & $-2.4 \pm 6.1$ & $73.0 \pm 10.5$ & $0.85 \pm 0.07$ & $-2.0 \pm 3.2$ & $135.0 \pm 24.3$ \\
$35 \%$ MDS & $0.0 \pm 0.0$ & $75.4 \pm 0.0$ & $1.00 \pm 0.00$ & $0.0 \pm 0.0$ & N/A \\
$40 \%$ MDS & $21.9 \pm 8.3$ & $97.3 \pm 14.0$ & $1.14 \pm 0.12$ & $12.9 \pm 3.1$ & N/A \\
$50 \%$ MDS & $46.2 \pm 11.2$ & $121.6 \pm 17$ & $1.42 \pm 0.12$ & $27.3 \pm 2.4$ & N/A \\
$60 \%$ MDS & $70.6 \pm 14.4$ & $146.0 \pm 21.0$ & $1.70 \pm 0.14$ & $41.7 \pm 1.9$ & N/A \\
\hline
\end{tabular}

Values are reported as mean $\pm S D$.

$\mathrm{CMJ}=$ countermovement vertical jump; $\mathrm{BW}=$ body weight factor; $1 \mathrm{RM}=1$-repetition maximum; $\mathrm{MDS}=$ maximal dynamic strength

Negative values represent unloading masses. 35\% MDS represents the body mass condition.

Calculations:

MDS $=1 \mathrm{RM}+[$ Body mass - Shank mass $]$

Un/Ext mass $=[$ MDS $\times \%$ MDS $]-[$ Body mass - Shank mass $]$;

$\mathrm{CMJ}$ load $=[$ Body mass - Shank mass $]+$ Un/Ext mass;

$\mathrm{BW}=\mathrm{CMJ}$ load $\div$ Body mass;

$\% 1 \mathrm{RM}=[$ Un/Ext mass $\div 1 \mathrm{RM}] \times 100$.

that must also be lifted (7,9). Cormie et al. (7) have previously calculated MDS with the shank mass equal to $12 \%$ of total body mass. In the current investigation, loads equal to body mass, $10,20,30,40,50$, and $60 \%$ of MDS were used. The body mass minus the shank mass was then subtracted from the \%MDS value to determine a negative unloading mass that was added to the unloading apparatus, or a positive external mass that was added in the form of a barbell and weight plates. To make direct comparisons with past investigations that have used \%1RM $(1,2,7,16,20)$ and body-weight factor $(3,15)$ methodology for determining the CMJ loading spectrum, the \%MDS loads were later expressed as \%1RM and body-weight factors based on the average loads used in both the trained (Table 2) and untrained groups (Table 3).

Unloading Apparatus. To test the effects of unloading on CMJ power, a custom-designed cable-pulley system was designed (Figure 1). Subjects were tightly secured into a harness that was attached to a cable-pulley system. To quantify the

TABLE 3. CMJ loading and unloading conditions for the untrained group.

\begin{tabular}{lccccc}
\hline & Un/Ext mass $(\mathrm{kg})$ & CMJ load $(\mathrm{kg})$ & BW & \%1RM & Unload force (N) \\
\hline 10\% MDS & $-52.2 \pm 14.5$ & $13.4 \pm 2.7$ & $0.18 \pm 0.02$ & $-76.8 \pm 18.1$ & $467.4 \pm 272.7$ \\
$20 \%$ MDS & $-38.8 \pm 12.1$ & $26.8 \pm 5.4$ & $0.36 \pm 0.04$ & $-57.1 \pm 15.8$ & $387.1 \pm 114.0$ \\
$30 \%$ MDS & $-25.4 \pm 9.9$ & $40.2 \pm 8.1$ & $0.55 \pm 0.06$ & $-37.6 \pm 13.9$ & $283.0 \pm 94.0$ \\
$40 \%$ MDS & $-12.0 \pm 8.0$ & $53.6 \pm 10.8$ & $0.73 \pm 0.07$ & $-18.0 \pm 11.3$ & $189.0 \pm 59.9$ \\
$50 \%$ MDS & $1.4 \pm 6.6^{*}$ & $67.1 \pm 13.5$ & $0.91 \pm 0.09$ & $1.9 \pm 10.7$ & N/A \\
$56 \%$ MDS & $0.0 \pm 0.0$ & $65.6 \pm 0.0$ & $1.00 \pm 0.00$ & $0.0 \pm 0.0$ & N/A \\
$60 \%$ MDS & $14.9 \pm 6.2$ & $80.5 \pm 16.2$ & $1.09 \pm 0.11$ & $20.9 \pm 8.9$ & N/A \\
\hline
\end{tabular}

Values are reported as mean $\pm S D$. CMJ = countermovement vertical jump; $\mathrm{BW}=$ body weight factor; $1 \mathrm{RM}=1$-repetition maximum; MDS = maximal dynamic strength. Negative values represent unloading masses.

*Based on the MDS calculation, some untrained subjects were loaded and others unloaded at the 50\% MDS condition. It is important to note that this value simply represents the average load used for this condition.

Calculations:

$\mathrm{MDS}=1 \mathrm{RM}+[$ Body mass - Shank mass $]$

Un/Ext mass $=[\mathrm{MDS} \times \% \mathrm{MDS}]-[$ Body mass - Shank mass $]$

$\mathrm{CMJ}$ load $=[$ Body mass - Shank mass $]+$ Un/Ext mass;

$\mathrm{BW}=\mathrm{CMJ}$ load $\div$ Body mass;

$\% 1 \mathrm{RM}=[$ Un/Ext mass $\div 1 \mathrm{RM}] \times 100$. 


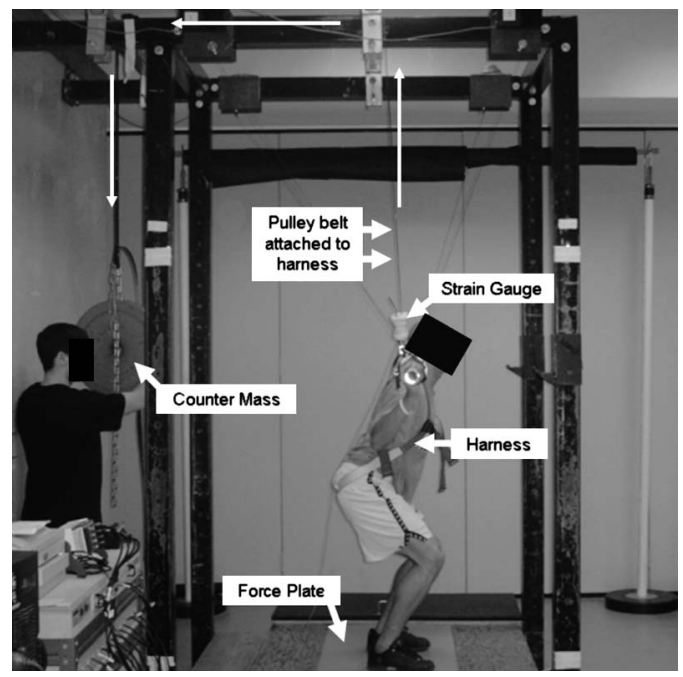

Figure 1. Unloading apparatus.

amount of unloading force, a strain gauge force transducer was positioned into the system. Because of the ability of subjects to accelerate vertically at a rate greater than that of the unloading mass accelerating downward, a single test administrator implemented manual unloading during all trials by providing a constant tension on the load to prevent slack in the cable. To ensure that the desired level of unloading was attained, data were visually monitored on a computer screen throughout the testing procedures and then later analyzed after testing. The average unloading forces collected from the strain gauge force transducer during the CMJ trials are reported in Tables 2 and 3 for the trained and untrained groups, respectively.

Measurement of Power. The methods used for collecting and analyzing CMJ performance have been validated and described previously $(4-7,18)$. During the jumps, subjects were required to hold a bar across their shoulders and keep a constant downward pressure on the bar so that it would not move independently of the body. For unloading trials and the jump at body mass, subjects used a minimally weighted plastic bar. For trials involving an additional load, a weighted bar and weight plates were used. Attached to the bar were 2 linear position transducers (Celesco Transducer Products, PT5A-150, Chatsworth, CA, USA) that were mounted on top of the rack, anterior and posterior to the subject. Combining trigonometry using known displacements and the displacement measurements from the position transducers, vertical displacement, and velocity were measured. Signals from the 2 position transducers and the force plate underwent rectangular smoothing with a moving average half-width of 12. The analog signals were collected at 1,000 $\mathrm{Hz}$ using a BNC-2010 interface box with an analog-to-digital card (National Instruments, NI PCI-6014, Austin, TX, USA). Custom-designed LabVIEW (National Instruments, Version 8.2) programs were used to collect and analyze the data. Peak and mean power were assessed during the concentric phase of the CMJs. Power-time curves were generated by multiplying force-time curves and velocity-time curves. Peak power was the maximal power recording from the power-time curve. Mean power was determined by dividing the total power produced over the concentric phase by the time it took to complete the concentric phase.

\section{Statistical Analyses}

Descriptive data were summarized as mean $\pm S D$. Intraclass correlation coefficients for reliability of the unloading forces and CMJ power were assessed using Cronbach's alpha. Differences between subject characteristics of the trained and untrained groups were determined using independent $t$-tests with a criterion alpha level of $p \leq 0.05$. Differences in power output across the loading and unloading spectrum, in comparison to the body mass condition, were determined for each group using a 1-way repeated measures analysis of

TABLE 4. Power output in the trained group across the loading and unloading spectrum.

\begin{tabular}{lllccrr}
\hline & CMJ PP $(W)$ & ES & Power & CMJ MP $(W)$ & ES & Power \\
\hline $10 \%$ MDS & $4,217 \pm 672^{*}$ & 2.50 & 1.00 & $2,117 \pm 402^{*}$ & 3.74 & 1.00 \\
$20 \%$ MDS & $5,048 \pm 1,292^{*}$ & 1.08 & 0.79 & $2,592 \pm 679^{\star}$ & 2.17 & 1.00 \\
$30 \%$ MDS & $5,949 \pm 1,168^{*}$ & 0.31 & 0.12 & $3,372 \pm 704^{*}$ & 0.90 & 0.63 \\
$35 \%$ MDS & $6,274 \pm 948$ & N/A & N/A & $3,949 \pm 565$ & N/A & N/A \\
$40 \%$ MDS & $5,894 \pm 803^{*}$ & 0.43 & 0.20 & $3,559 \pm 457^{\star}$ & 0.76 & 0.49 \\
$50 \%$ MDS & $5,439 \pm 733^{*}$ & 0.99 & 0.71 & $3,105 \pm 352^{*}$ & 1.79 & 1.00 \\
$60 \%$ MDS & $5,274 \pm 772^{*}$ & 1.16 & 0.84 & $2,863 \pm 394^{*}$ & 2.23 & 1.00 \\
\hline
\end{tabular}

$\mathrm{CMJ} \mathrm{PP}=$ countermovement jump peak power; $\mathrm{ES}=$ effect size; $\mathrm{CMJ} \mathrm{MP}=$ countermovement jump mean power; $\mathrm{MDS}=$ maximal dynamic strength.

*Significant difference $(p \leq 0.05)$ from 35\% MDS (body mass) loading condition. 
TABLE 5. Power output in the untrained group across the loading and unloading spectrum.

\begin{tabular}{lcccccc}
\hline & CMJ PP $(W)$ & ES & Power & CMJ MP $(W)$ & ES & Power \\
\hline $10 \%$ MDS & $2,217 \pm 207^{*}$ & 2.21 & 0.93 & $1,200 \pm 65^{*}$ & 2.80 & 0.99 \\
$20 \%$ MDS & $2,303 \pm 451^{*}$ & 1.88 & 0.84 & $1,207 \pm 257^{*}$ & 2.40 & 0.96 \\
$30 \%$ MDS & $2,632 \pm 412^{*}$ & 1.42 & 0.60 & $1,414 \pm 107^{*}$ & 2.05 & 0.89 \\
$40 \%$ MDS & $2,796 \pm 677$ & 1.01 & 0.35 & $1,555 \pm 372^{*}$ & 1.24 & 0.49 \\
$50 \%$ MDS & $3,210 \pm 707$ & 0.47 & 0.11 & $1,854 \pm 443$ & 0.45 & 0.11 \\
$56 \%$ MDS & $3,571 \pm 842$ & N/A & N/A & $2,047 \pm 423$ & N/A & N/A \\
$60 \%$ MDS & $3,151 \pm 719$ & 0.94 & 0.31 & $1,710 \pm 278^{*}$ & 0.94 & 0.31 \\
\hline
\end{tabular}

$\mathrm{CMJ} \mathrm{PP}=$ countermovement jump peak power; $\mathrm{ES}=$ effect size; $\mathrm{CMJ} \mathrm{MP}=$ countermovement jump mean power; $\mathrm{MDS}=$ maximal dynamic strength.

*Significant difference ( $p \leq 0.05$ ) from 56\% MDS (body mass) loading condition.

variance followed by a Holm's sequential Bonferroni procedure to determine pairwise differences. Because the purpose of the current study was to test the MDO hypothesis (i.e., if power at body mass was different than at the 6 other loading conditions), the number of post hoc comparisons was limited to 6 (body mass vs. 10, 20, 30, 40, 50, and $60 \%$ MDS). Because of the extensive number of potential pairwise comparisons that can be made among 7 loading conditions, the number of comparisons was limited to 6 to prevent deflation of the corrected alpha level and to eliminate comparisons that would be irrelevant to the study purpose. Additionally, meaningfulness of the differences from power output during the various loading conditions compared with power output during the body mass condition was determined by the use of effect sizes. Effect size (Cohen's $d$ ) was calculated as the difference in the mean values, divided by the pooled $S D$ (21). Furthermore, a post hoc statistical power analysis $\left(G^{*}\right.$ Power 3.0.10) (10) was completed to assess the likelihood of detecting real differences. Statistical analyses were completed using a statistical software package (SPSS Version 15.0, SPSS Inc., Chicago, IL, USA).

\section{RESUlts}

\section{Reliability}

The average unloading forces for the trained group were consistent as $r=0.87$ (10\% MDS), $r=0.91$ (20\% MDS), and $r=0.88$ (30\% MDS). The average unloading forces for the untrained group were consistent as $r=0.99$ (10\% MDS), $r=$ 0.99 (20\% MDS), $r=0.99$ (30\% MDS), and $r=0.97(40 \%$ MDS). The measurement of CMJ power was also consistent throughout testing as $r=0.96$ in the trained group and $r=$ 0.94 in the untrained group.

\section{Countermovement Vertical Jumping Power}

For both the trained and untrained groups, peak and mean power output were greatest during the body mass jump condition and were significantly different from various conditions of loading and unloading. For the trained group, these data can be found in Table 4. For the untrained group, these data can be found in Table 5 .

\section{Discussion}

The purpose of this investigation was to assess the MDO hypothesis in 2 distinct subject groups to determine if levels of maximal strength affect the load that maximizes CMJ power output. It was discovered that both trained and untrained group averages for CMJ peak and mean power output were maximal when jumping at body mass. Thus, significant differences in maximal strength levels between the trained and untrained groups in this study did not affect the load that maximized CMJ power output. These findings support the MDO hypothesis in subjects with significantly different levels of lower-body strength.

The MDO hypothesis has been developed from evidence, which has indicated that when valid measurement techniques and comprehensive loading spectrums are used in assessing the CMJ load-power relationship, power output is maximized at body mass $(3,7,8,15,16)$. The MDO hypothesis states that "the muscular system of the lower limbs is designed to produce maximum dynamic output in rapid movements when loaded only by the weight and the inertia of its own body" (13). The findings from the current investigation support the MDO hypothesis as both the trained and untrained groups, who demonstrated significant differences in 1RM-to-body mass ratios, maximized $\mathrm{CMJ}$ peak and mean power output at body mass. The finding of decreased mean power as a result of both loading and unloading is consistent with the findings by Markovic and Jaric (13). In the current investigation, peak power was also decreased as a result of loading, and this is also consistent with previous findings $(7,15)$. However, the current investigation observed a decrease in peak power when unloading occurred and these results conflict with the findings by Markovic and Jaric (15) who observed essentially no change in peak power with unloading. It is currently 
unclear as to why the effects of unloading on peak power were different between the current investigation and the work by Markovic and Jaric (15). One possible explanation for these varied results may be the type of unloading used and the subsequent effect on peak velocity. In the current investigation, unloading was accomplished by hanging a countermass onto a cable-pulley system, and during conditions of unloading, peak velocity either decreased or remained unchanged. However, Markovic and Jaric (15) used an unloading technique that incorporated elastic band tension, and during conditions of unloading, they observed increased peak velocities. Thus, the different designs of the unloading apparatus may have ultimately affected the attainment of peak velocity and peak power values during the CMJ unloading. The length-tension relationship of the elastic bands may provide a change in unloading force throughout the CMJ concentric phase, which optimally matches the change in the velocity of muscle contraction. Consequently, the attainment of higher peak velocities may result. It appears that establishing a standardized CMJ unloading technique will be of importance for future research regarding the MDO hypothesis.

Previous findings have indicated potential differences in the load that maximizes power output depending on levels of lower-body maximal strength $(1,20)$, thus negating the application of the MDO hypothesis to all populations. For example, Stone et al. (20) demonstrated that trained subjects, who had high 1RM-to-body mass ratios (2.00) as a result of extensive strength and power training, were able to further load their body mass up to a load of $40 \% 1 \mathrm{RMto}$ maximize power, whereas untrained subjects with low 1RM-to-body mass ratio (1.21) attained maximal power output at the lightest load studied (10\% 1RM). However, findings from the current investigation do not support the idea that differences in lower-body maximal strength affect the load which maximizes power output. The reason for these conflicting results may be related to the absolute strength of the strength-power trained subjects and their past training experiences. In the current investigation, trained subjects had an average squat $1 \mathrm{RM}$ of $168 \mathrm{~kg}$ and the majority participated in body-weight sports (e.g., baseball, basketball, track and field sprinting and jumping, or volleyball). However, the average squat 1RM of the highly trained subjects reported by Stone et al. (20) was $213 \mathrm{~kg}$. Coincidentally, the only trained subject in the current investigation to have a squat $1 \mathrm{RM}$ greater than $213 \mathrm{~kg}$ was a competitive powerlifter who also demonstrated greater power outputs when externally loaded and not at body mass. It should also be noted that this subject had a high 1RM-tobody mass ratio of 2.42. Furthermore, the strength-power trained subjects in this study were also good vertical jumpers (average CMJ height of $0.55 \mathrm{~m}$ ), especially when considering that the method used to assess CMJ height did not permit an arm swing. Thus, it could be argued that one limitation to the current study was the selection bias of strength-power subjects who were clearly already skilled at jumping with their own body weight. Thus, future investigation may be warranted to determine (a) if the MDO hypothesis applies to elite level strength-power athletes (e.g., powerlifters and Olympic weightlifters) who have 1RM-to-body mass ratios greater than 2.00 and who are continually exposed to heavy external masses via daily heavy resistance training, and (b) if the MDO hypothesis applies to subjects who are both strong and skilled at jumping at body mass vs. subjects who are strong but unskilled at jumping at body mass.

Another point that warrants discussion regarding the MDO hypothesis is the potential effect of body composition on CMJ power output at body mass. In the current investigation, the untrained group had a percentage of body fat $(14.4 \%)$ that was significantly greater than that of the trained group $(8.6 \%)$. It is interesting to consider what may happen to $\mathrm{CMJ}$ power at body mass in the untrained group if, via a health intervention, body fat percentage is reduced, possibly to a level similar to that of the trained group, while lower-body maximal strength is maintained. Theoretically, this type of situation should have been mimicked in the current study during trials of unloading because both unloading masses and fat mass represent inert masses. In the current study, it was discovered that unloading decreased $\mathrm{CMJ}$ power output. However, it is unlikely that a decrease in CMJ power output at body mass would be observed after participation in a health intervention that decreases body fat percentage and maintains lower-body strength. In fact, CMJ power output at body mass would likely increase in such a situation because of an increase in CMJ velocity. Thus, it may be implied that the body's neuromuscular system is continually adapting throughout such interventions to be most powerful at body mass, and that using unloading to mimic situations of fat loss is not appropriate because it does not account for such neuromuscular adaptations. Therefore, unloaded CMJs should only be interpreted in their true biomechanical sense, which is an acute and temporary situation of microgravity. Additional consideration should be given to untrained subjects who are obese and who have much greater body fat percentages than those reported in the current study (e.g., 30\%). At this point, it is unclear as to what happens to CMJ power in obese persons during situations of unloading because no studies have assessed unloaded CMJs in this population. If the MDO hypothesis holds true across all populations, even obese persons with high body fat percentages will maximize $\mathrm{CMJ}$ power output at body mass rather than in situations of unloading.

In the current study, loads were determined as a percentage of MDS and then later expressed as a percentage of the 1RM and body-weight factors so that the results from the current study can be easily compared with previous studies which have used these methodologies. Determining CMJ loads as a percentage of MDS is important because the MDS calculation accounts for the external load lifted during the $1 \mathrm{RM}$ test in addition to the individual body mass minus the shanks that must also be lifted during the 1RM test. The use of 
Journal of Strength and Conditioning Research" | www.nsca-jscr.org

MDS also helps to establish comparable loading spectrums for subjects with varied squat $1 \mathrm{RM}$-to-body mass ratios, and the MDS calculation may also help put the MDO hypothesis in context with other body movements such as elbow flexion. Previously, it has been reported that in the elbow flexion movement, power is maximized at a load equal to $30-35 \%$ of maximal strength (23). In the current study, both trained and untrained subjects generated maximal power during the body mass jump condition; however, the body mass condition did not necessarily correspond to a load equal to $30-35 \%$ of MDS in both groups. In the trained group, the body mass load was equal to $35 \%$ of MDS; however, in the untrained group, the body mass load was equal to $56 \%$ of MDS. Therefore, it appears that in the CMJ, trained subjects maximize CMJ power at a similar load to that which has been reported previously in single-joint movements (23). On the other hand, it appears that untrained individuals do not maximize CMJ power at $30 \%$ of MDS because this condition requires that they be unloaded because of their low 1RM-to-body mass ratios. Thus, because of the significantly different 1RMto-body mass ratios between the 2 groups, the body mass condition corresponded to a load equal to $35 \%$ MDS in the trained group and 56\% MDS in the untrained group.

In conclusion, the current investigation demonstrated that CMJ power output was maximized when jumping at body mass in both trained and untrained subjects who had significantly different $1 \mathrm{RM}$-to-body mass ratios. However, because of the significant differences in 1RM-to-body mass ratios, the body mass condition corresponded to a load equal to $35 \%$ of MDS in the trained group and 56\% of MDS in the untrained group. These findings help clarify the CMJ loadpower relationship, and in addition, support the MDO hypothesis.

\section{Practical Applications}

Identification of the optimal load for power has practical significance for those who seek to design training programs that may improve power capabilities. Previously, using the biceps model, it was discovered that training at the optimal load for power was the most effective training method for increasing overall power output (14). However, since that time, new evidence from the same authors has demonstrated that the most effective way to increase overall power capabilities is not by training solely with the load that maximizes power but by training at the optimal load in addition to loads across the entire loading spectrum (i.e., strength-power training) $(22,24)$. More recently, using the jump squat model, the effectiveness of combined strength (back squats at 90\% 1RM) and power training (body mass jump squats) has been found to be a superior method for overall power development than simply training at the optimal load (6). The current study provides further, indirect support for the use of body mass jumps in power training programs for both trained and untrained individuals because of the maximal power outputs that are produced at this load.
However, it is strongly recommended that lower-body resistance training with loads across the loading spectrum occur concurrently with body mass CMJs to receive optimal power adaptations.

\section{Acknowledgments}

This study was supported through funding by the National Strength and Conditioning Association's Graduate Research Grant.

\section{References}

1. Baker, D. A series of studies on the training of high-intensity muscle power in rugby league football players. J Strength Cond Res 15: 98-209, 2001.

2. Baker, D, Nance, S, and Moore, M. The load that maximizes the average mechanical power output during jump squats in powertrained athletes. J Strength Cond Res 15: 92-97, 2001.

3. Cavagna, GA, Zamboni, A, Faraggiana, T, and Margaria, R. Jumping on the moon: Power output at different gravity values. Aerosp Med 43: 408-414, 1972.

4. Cormie, P, Deane, R, and McBride, JM. Methodological concerns for determining power output in the jump squat. J Strength Cond Res 21: 424-430, 2007.

5. Cormie, P, McBride, JM, and McCaulley, GO. Validation of power measurement techniques in dynamic lower body resistance exercises. J Appl Biomech 23: 103-118, 2007.

6. Cormie, P, McCaulley, GO, and McBride, JM. Power versus strength-power jump squat training: influence on the load-power relationship. Med Sci Sports Exerc 39: 996-1003, 2007.

7. Cormie, P, McCaulley, GO, Triplett, NT, and McBride, JM. Optimal loading for maximal power output during lower-body resistance exercises. Med Sci Sports Exerc 39: 340-349, 2007.

8. Davies, CT and Young, K. Effects of external loading on short term power output in children and young male adults. Eur J Appl Physiol Occup Physiol 52: 351-354, 1984.

9. Dugan, EL, Doyle, TLA, Humphries, B, Hasson, CJ, and Newton, RU. Determining the optimal load for jump squats: A review of methods and calculations. J Strength Cond Res 18: 668-674, 2004.

10. Faul, F, Erdfelder, E, Lang, A, and Buchner, A. G*Power 3: A flexible statistical power analysis program for the social, behavioral, and biomedical sciences. Behav Res Methods 39: 175-191, 2007.

11. Hori, N, Newton, RU, Andrews, WA, Kawamori, N, McGuigan, MR, and Nosaka, K. Comparison of four different methods to measure power output during the hang power clean and weighted squat jump. J Strength Cond Res 21: 314-320, 2007.

12. Jackson, AS and Pollock, ML. Generalized equations for predicting body density for men. Br J Nutr 40: 497-504, 1978.

13. Jaric, $\mathrm{S}$ and Markovic, G. Leg muscles design: the maximum dynamic output hypothesis. Med Sci Sports Exerc 41: 780-787, 2009.

14. Kaneko, M, Fuchimoto, T, Toji, H, and Suei, K. Training effect of different loads on the force-velocity relationship and mechanical power output in human muscle. Scand J Sports Sci 5: 50-55, 1983.

15. Markovic, $\mathrm{G}$ and Jaric, S. Positive and negative loading and mechanical output in maximum vertical jumping. Med Sci Sports Exerc 39: 1757-1764, 2007.

16. McBride, JM, Triplett-McBride, T, Davie, A, and Newton, RU. A comparison of strength and power characteristics between power lifters, Olympic lifters, and sprinters. J Strength Cond Res 13: 58-66, 1999.

17. McBride, JM, Triplett-McBride, T, Davie, A, and Newton, RU. The effect of heavy- vs. light-load jump squats on the development of strength, power, and speed. J Strength Cond Res 16: 75-82, 2002. 
18. McCaulley, GO, Cormie, P, Cavill, MJ, Nuzzo, JL, Urbiztondo, ZG and McBride, JM. Mechanical efficiency during repetitive vertical jumping. Eur J Appl Physiol 101: 115-123, 2007.

19. Siri, W. Body composition from fluid spaces and density: Analysis of methods. In: Techniques for Measuring Body Composition. Brozek, J and Henschel, A, eds. Washington, DC: National Academy of Science, 1961. pp. 223-244.

20. Stone, MH, O’Bryant, HS, McCoy, L, Coglianese, R, Lehmkuhl, M, and Schilling, B. Power and maximum strength relationships during performance of dynamic and static weighted jumps. J Strength Cond Res 17: 140-147, 2003
21. Thomas, JR, Nelson, JK, and Silverman, SJ. Research Methods in Physical Aactivity (5th ed.). Champaign, IL: Human Kinetics, 2005.

22. Toji, $\mathrm{H}$ and Kaneko, M. Effect of multiple-load training on the forcevelocity relationship. J Strength Cond Res 18: 792-795, 2004.

23. Toji, $\mathrm{H}$ and Kaneko, $\mathrm{M}$. Effects of aging on force, velocity, and power in the elbow flexors of males. J Physiol Anthropol 26: 57-592, 2007.

24. Toji, H, Suei, K, and Kaneko, M. Effects of combined training loads on relations among force, velocity, and power development. Can J Appl Physiol 22: 328-336, 1997. 